\title{
A Robust Optimization for Multi-Period Lost Sales Inventory Control Problem
}

\author{
Shunichi Ohmori \\ Department of Industrial and Management System Engineering, \\ Waseda University, Okubo 3-4-1, Shinjuku, Tokyo 169-8555 Japan \\ E-mail: ohmori0406@aoni.waseda.jp \\ Kazuho Yoshimoto \\ Department of Industrial and Management System Engineering, \\ Waseda University, Okubo 3-4-1, Shinjuku, Tokyo 169-8555, Japan \\ E-mail: kazuho@waseda.jp
}

\begin{abstract}
We consider a periodic review inventory control problem of minimizing inventory cost, production cost, and lost sales under demand uncertainty, in which product demands are not specified exactly and it is only known to belong to a given uncertainty set. We propose a robust optimization formulation for obtaining lowest cost possible and guaranteeing the feasibility with respect to range of order quantity and inventory level for possible values of the data from the uncertainty set. Our formulation is based on the affinely adaptive robust counterpart, which suppose order quantity is affine function of past demands. We derive certainty equivalent problem via second-order cone programming, which gives 'not too pessimistic' worst-case.
\end{abstract}

Keywords: adaptive robust, optimization, inventory control, second order-cone-programming

\section{INTRODUCTION}

We consider the problem of finding a robust policy to control the inventory level under demand uncertainty. Inventory control is an essential problem and has been extensively studied in the operations research for over many decades. There are two approaches when inventory is short and the demand is not satisfied, lost-sales model, and backorder model. In lost-sales model, the excess demands are lost, whereas in the backorder model, customer will wait for the stock to be replenished. In this paper, we consider the lost-sales inventory control problem. The lost-sales setting can often be seen in many retail environments, and the importance of the model is reasoned by Gruen et al. (2002) revealing that only $15 \%$ of the customers who observe a stock out will delay the purchase, and the other $85 \%$ of customers will not buy the product at the store. Especially in grocery or apparel industry, it is claimed that 60 to 70 percent of purchases are unplanned (Underhill 2009). Clearly, stock out can lead to lost sales as well as disappointment and frustration for customers. These facts make the lost-sales model an important topic. The survey research on this topic is conducted by Bijvank and Vis (2011). The main focus of this topic has been considering optimal or suboptimal ordering policy for infinite time horizon. Early research on the optimal policy include Bellman et al. (1955) and Karlin and Scarf (1958), where lead time is assumed to be one review period. Morton (1969) extends these models to a general non-zero lead time problem. Zipkin (2008) reformulated the model of Karlin (1958) and Morton (1969) and analyze the structure of the problem using discrete convex analysis. There are also lots of suboptimal policies for different problem settings such as (Gaver 1959, Morse 1959, Pressman 1977, Van Donselaar 1996, Johansen 2001, Downs 2001, Johansen 2001, Janakiraman 2004, Huh 2009). All these models assume that demands are independent and identically distributed i.i.d using Gaussian or Poisson, and few papers model demand distribution with different parameters or correlations between periods. The need to model demand distribution with different parameters between periods arises in several applications, for example, when there are specific forecasts of demands for each period on a short-term basis. Such a setting can often be seen more in the literatures of dynamic lot-sizing problem under demand uncertainty. See Tempelmeier (2013) and Aloulou et al. (2014) for review. These models are finite horizon models and differ from infinite horizon models in that the demand distributions for each period are given and try to minimize some cost function, such as production cost, inventory cost, as well as lost sales. Typical approach is scenario-based approach such as Guan et al. (2006) and Zhou and Guan (2013) and solve the problem by dynamic programming (DP). Although DP is a very powerful approach and works very well for small sized problem, the number of intermediate states used during computation grows exponentially as the number of variables increase, known as "curse of dimensionality". Therefore, this approach is not applicable for when the problem size becomes large. Another approach is setting order-up-to level during successive replenishment periods such as Tarim and Kingsman (2004), Tunc et al. (2014) via mixed integer programming (MIP). This modelling also includes integer variables and thus are not applicable for larger problems.

Robust optimization has been drawing attention recently as an alternative way to deal with demand uncertainty in the inventory control problem. It can incorporate demand uncertainty into optimization models, in which product demands are not specified exactly and it is only known to belong to a given uncertainty set, yet the 
worst-case total cost is minimized while satisfying constraints for all possible values of the data from the uncertainty set. The benefit of the modelling is that problems can be reduced to equivalent deterministic problems, such as linear programming (LP) or second-order cone programming (SOCP), and thus can be solved very fast using efficient algorithms for these problem classes. There are several applications of robust optimization to inventory control problem. Bertsimas and Sim (2003) and Bertsimas and Thiele (2004) applied robust optimization to finite-horizon multi-period inventory control problem. They model the demand uncertainty set using budge of uncertainty robust (BUR) formulation proposed by Bertsimas and Thiele (2006), in which degree of robustness and conservatism are controlled by the scaled deviation of parameters from their nominal values. They proposed LP based model for problem without fixed ordering cost, and MIP based model for problem with fixed ordering cost. Bienstock (2008) studied the optimal base stock level in the same model and proposed algorithms based on decomposition. Another stream of formulation via robust optimization is affinely adjustable robust counterpart (AARC) formulation proposed by BenTal et al. (2004), in which part of the decision variables are assumed to be determined after a portion of the uncertain data is realized and those adjustable variables are modelled to be affine functions of uncertain data. In inventory control context, order quantity can be determined based on the realization of past demands. Ben-Tal et al. (2009) studied retailer-supplier flexible commitment problem with uncertain demand, and Ben-Tal et al. (2005) studied multiechelon inventory control problem, both using AARC. See and Sim (2010) proposed approximate objective function formulation considering non-zero lead time and demand is a factor based. Solyal et al. (2016) proposed a formulation disaggregating order quantity for the demand of each period by specifying when to place an order to satisfy the demand of a specific period and proposed efficient heuristics to solve the problem.

Even with the progress, however, all of these models are inventory control with backorder model, where all of excess demands are backlogged, and to our best knowledge, no robust optimization model has been proposed to lost-sales inventory control problem. In this paper, we propose a robust optimization formulation for lost-sales inventory control problem in which excess demands are lost. Our formulation is based on the AARC, which suppose order quantity is affine function of past demands. We derive certainty equivalent problem via SOCP, which gives 'not too pessimistic worst-case'. The outline of the paper is as follows. In section 2, we describe the basic lost-sales inventory control problem, formulated as non-robust models. In section 3, we derive robust models for the problem described in section 2. In section 4, we give numerical results, and in section 5 , we present a conclusion and future work.

The reminder of this paper is as follows. In section 2, we review the existing literatures. In section 3 , we explain our proposed model. In section 4 , we describe the field data used to estimate our model. We then discuss our results and managerial implications based on the behavioural findings. In section 5, we provide conclusion.

\section{NON-ROBUST LOST-SALES INVENTORY CONTROL PROBLEM}

In this section, we present mathematical formulations of non-robust lost-sales inventory control problem. First, we give a standard non-robust problem including equality and inequality constraints. Then, we eliminate equality constraints from the original problem so as to apply robust optimization and reformulate the problem in a vector-matrix form. Finally, we impose constraints that order quantity should be affine function of the past demand history to apply AARC.

\subsection{Floor Representation} follows

The lost-sales inventory control problem is presented as

$$
\begin{aligned}
& \operatorname{minimize} \quad \sum_{t=1}^{T}\left(h_{t} x_{t}+c_{t} u_{t}+p_{t} s_{t}\right) \\
& \text { subject to } \quad x_{t}=x_{t-1}+u_{t}-d_{t}+s_{t} \\
& \quad(t=1, \cdots, T) \\
& \underline{u}_{t} \leq u_{t} \leq \bar{u}_{t}(t=1, \cdots, T) \\
& \underline{x}_{t} \leq x_{t} \leq \bar{x}_{t}(t=1, \cdots, T) \\
& s_{t} \geq 0 \quad(t=1, \cdots, T)
\end{aligned}
$$

Decision variable

- $s_{t}$ : shortage (amount of unmet demand) at time $t$

- $x_{t}$ : inventory level at time $t$

- $u_{t}$ : order quantity at time $t$

\section{Parameters}

- $p_{t}$ : Price of product at time $t$

- $c_{t}$ : Unit production cost at time $t$

- $h_{t}$ : Unit inventory holding cost at time $t$

- $d_{t}$ : Forecast of demand at time $t$

- $\underline{x}_{t}, \bar{x}_{t}$ : under and lower limit for inventory at time $t$

- $\underline{u}_{t}, \bar{u}_{t}$ : under and lower limit for order quantity at time $t$

- $x_{0}$ : initial inventory

The objective function is minimizing sum of inventory cost, production cost, and lost sales. Note that this function is same as profit maximization

$\operatorname{maximize} \quad \sum_{t=1}^{T} p_{t} d_{t}-\sum_{t=1}^{T}\left(h_{t} x_{t}+c_{t} u_{t}+p_{t} s_{t}\right)$

where sales term $p_{t} d_{t}$ is constant. Constraints (1b) present inventory update equation, in which all excess demands are lost. Constraints $(1 \mathrm{c}, 1 \mathrm{~d})$ requires order quantity $u_{t}$ and inventory $x_{t}$ are bounded.

This problem is a linear-programming and the optimal solution can be obtained using standard-solver. For the special case where $x_{t}$ and $u_{t}$ are not bounded, i.e., $x_{t}=$ 
$0, x_{t}=+\infty, u_{t}=0, u_{t}=+\infty$, optimal solution can be obtained analytically as $u_{t}=d_{t}, x_{t}=0, s_{t}=0$.

\subsection{Lost Sales Inventory Control Problem in Inequality Form}

In robust optimization, uncertain parameter can take all possible values within a given uncertainty set, so satisfying equality constraints including uncertain parameter is impossible. Therefore, equality constraints must be transformed to a set of inequality constraints.

Inventory update constraints (1b) can be transformed by backward substitution as follows:

$$
\begin{aligned}
x_{t} & =x_{t-1}+u_{t}-d_{t}+s_{t} \\
& =\left(x_{t-2}+u_{t-1}-d_{t-1}+s_{t-1}\right)+u_{t}-d_{t-1}+s_{t} \\
& \vdots \\
& =x_{0}+\sum_{i=1}^{t}\left(u_{i}-d_{i}+s_{i}\right)
\end{aligned}
$$

We can also express this equality using matrix form as

$$
\left[\begin{array}{c}
x_{1} \\
x_{2} \\
\vdots \\
x_{T}
\end{array}\right]=\left[\begin{array}{c}
x_{0} \\
x_{0} \\
\vdots \\
x_{0}
\end{array}\right]+\left[\begin{array}{ccccc}
1 & 0 & 0 & \cdots & 0 \\
1 & 1 & 0 & \cdots & 0 \\
& & \vdots & & \\
1 & 1 & 1 & \cdots & 1
\end{array}\right]+\left[\begin{array}{c}
u_{1}-d_{1}+s_{1} \\
u_{2}-d_{2}+s_{2} \\
\vdots \\
u_{T}-d_{T}+s_{T}
\end{array}\right]
$$

or more compactly,

$$
x=x_{0} \mathbf{1}+L(u-d+s)
$$

where $\quad\left[x_{1}, \cdots, x_{T}\right]^{T}, u=\left[u_{1}, \cdots, u_{T}\right]^{T}, d=$ $\left[d_{1}, \cdots, d_{T}\right]^{T}, s=\left[s_{1}, \cdots, s_{T}\right]^{T}$ and 1 denotes the vector with all entries one, and $\mathrm{L}$ denotes lower triangular matrix with all entries one. Using this expression, we can reformulate the original problem using matrix form as

minimize $\quad h^{T} x+c^{T} u+p^{T} S$

subject to $\quad x=x_{0} \mathbf{1}+L(u-d+s)$

$\underline{u} \leq u \leq \bar{u}$

$\underline{x} \leq x \leq \bar{x}$

$s \geq 0$

By eliminating $\mathrm{x}$, we can reformulate the problem in an inequality form as follows.

minimize $\quad h^{T}\left[x_{0} \mathbf{1}+L(u-d+s)\right]+c^{T} u+p^{T} s$

subject to $\underline{u} \leq u \leq \bar{u}$ $\underline{x} \leq x_{0} \mathbf{1}+L(u-d+s) \leq \bar{x}$

$s \geq 0$

Note that we can consider non-zero lead-time case by substituting $u$ with

$u^{L T}=\left[\begin{array}{c}u_{-L T+1} \\ \vdots \\ u_{0} \\ u_{1} \\ \vdots \\ u_{T-L T}\end{array}\right]$

where $L T$ denotes lead-time, $\left[u_{-L T+1}, \cdots, u_{0}\right]$ are given constants, and $\left[u_{1}, \cdots, u_{T-L T}\right]$ are decision variables. Without loss of generality, we consider the zero-lead-time case hereinafter.

\subsection{Lost Sales Inventory Control Problem in Affinely Adjustable LP Form}

To apply adjustable robust linear programming, we present adjustable LP formulation. In multi-period problem, order quantity should be determined based on the observation of past demand history. Considering this feature, adjustable approach assumes order quantity at each period must be affine function of past demand history as follows

$u_{t}=\sum_{j=1}^{t} v_{t j} d_{j}+w_{t}$

We can express this equality using matrix form as

$\left[\begin{array}{c}u_{1} \\ u_{2} \\ \vdots \\ u_{T}\end{array}\right]=\left[\begin{array}{rrrr}v_{11} & 0 & \cdots & 0 \\ v_{21} & v_{22} & \cdots & 0 \\ & \vdots & & \\ v_{T 1} & v_{T 1} & \cdots & 0\end{array}\right]\left[\begin{array}{c}d_{1} \\ d_{2} \\ \vdots \\ d_{T}\end{array}\right]+\left[\begin{array}{c}w_{0} \\ w_{1} \\ \vdots \\ w_{T}\end{array}\right]$

or more compactly,

$u=V d+w$

where denote lower triangular matrix with entries $v_{t j}$, and $w=\left[w_{1}, \cdots, w_{T}\right]^{T}$. Also, for lighter appearance, by defining $Z=V-I$ with $I$ as identity matrix, we use the following expression

$$
\begin{aligned}
u-d & =(V-I) d+w \\
& =Z d+w
\end{aligned}
$$

By using this expression, the objective function can be reformulated as follows

$$
\begin{array}{ll}
\operatorname{minimize} & h^{T}\left[x_{0} \mathbf{1}+L(u-d+s)\right]+c^{T} u+p^{T} s \\
& =x_{0} h^{T} \mathbf{1}+h^{T} L(u-d)+c^{T} u \\
& +\left(h^{T} L+p^{T}\right) s
\end{array}
$$




$$
\begin{aligned}
& =x_{0} h^{T} \mathbf{1}+\left(h^{T} L Z+c^{T} V\right) d \\
& +\left(h^{T} L+c^{T}\right) w+\left(h^{T} L+p^{T}\right) s \\
& =h_{0}+z^{T} d+f^{T} w+q^{T} s
\end{aligned}
$$

where

$$
\begin{aligned}
& h_{0}=x_{0} h^{T} \mathbf{1} \\
& z=\left(h^{T} L Z+c^{T} V\right)^{T} \\
& f=\left(h^{T} L+c^{T}\right)^{T} \\
& q=\left(h^{T} L+p^{T}\right)^{T}
\end{aligned}
$$

and the problem can be reformulated as follows

$$
\begin{aligned}
& \operatorname{minimize} \quad h_{0}+z^{T} d+f^{T} w+q^{T} s \\
& \text { subject to } \quad \underline{u} \leq V d+w \leq \bar{u} \\
& \underline{x} \leq x_{0} \mathbf{1}+L(Z d+w+s) \leq \bar{x} \\
& s \geq 0
\end{aligned}
$$

Note that a set of decision variables $(x, u, s)$ are transformed to $(V, w, s)$. Note also that the optimal value of the problem is equivalent to the original problem (1a-1e) in the deterministic demand case, but not in the uncertain demand case.

\section{PROBLEM FORMULATION}

In this section, we derive robust formulation of the problem. First, we mention demand uncertainty set using ellipsoidal bound. Next, we present two robust models. First model is static robust model derived from the problem (2a$2 \mathrm{~d}$ ), and the second model is dynamic robust model derived from the problem (3a-3d).

\subsection{Ellipsoidal Bound for Demand Uncertainty}

We assume $d$ llies in a given ellisoid:

$d \in \varepsilon=\left\{\mu+R a \mid\|a\|_{2} \leq 1\right\}$

It is known that if $d$ can be supposed to be Gaussian random vectors with mean $\mu$ and covariance $\Sigma, R$ can be expressed as

$R=\sqrt{\Phi^{-1}(T, \gamma)} \Sigma^{1 / 2}$,

where $\Phi(n, \gamma)$ is the cumulative distribution function of $\chi^{2}$ distributed variable.

\subsection{Robust Static Model}

Let $\tilde{l}_{t}^{T}$ denote $t$-th row vector of lower triangular matrix $L$. The $t$-th row vector of the term $L d$ appeared in (2a, 2c) can be expressed as follows:

$$
\begin{aligned}
\max _{d \in \varepsilon}\left(\tilde{l}_{t}^{T} d\right) & =\tilde{l}_{t}^{T} u+\left\|\tilde{l}_{t}^{T} R e_{t}\right\|_{2} \\
& =\tilde{l}_{t}^{T} u+\Delta d_{t} \\
\min _{d \in \varepsilon}\left(\tilde{l}_{t}^{T} d\right) & =\tilde{l}_{t}^{T} u-\Delta d_{t}
\end{aligned}
$$

where $e_{t}$ denotes the vector with $t$-th standard basis vector ( $t$-th entry is one and all other entries are zero), $\Delta d_{t}=$ $\left\|\tilde{l}_{t}^{T} R e_{t}\right\|_{2}$. From (4), we can derive non-robust static model as

$\operatorname{minimize} \sum_{t=1}^{T} h\left(x_{0}+\tilde{l}_{t}^{T}(u-\mu+s)\right)+c_{t} u_{t}+p_{t} s_{t}$

subject to $\quad \underline{u}_{t} \leq u_{t} \leq \bar{u}_{t}(t=1, \cdots, T)$

$\underline{x}_{t} \leq x_{0}+\tilde{l}_{t}^{T}(u-\mu+s)-\Delta d_{t}$

$(t=1, \cdots, T)$

$x_{0}+\tilde{l}_{t}^{T}(u-\mu+s)+\Delta d_{t} \leq \bar{x}_{t}$

$(t=1, \cdots, T)$

$s_{t} \geq 0 \quad(t=1, \cdots, T)$

This problem is a LP and thus can be solved efficiently. Note that we did not add $\Delta d_{t}$ term in the objective function, because $\Delta d_{t}$ is a constant and does not depend on decision variables.

\subsection{Dynamic Robust Model}

Let $\tilde{l}_{t}^{T}$ denote $t$-th row vector of lowertriangular matrix $V, \tilde{z}_{t}^{T}$ denote $t$-th row vector of lower triangular matrix $Z$. Considering the minimum and maximum values for $z d, V, d, L Z d$ in the same way as in (4), we can derive robust static model as

$\operatorname{minimize} \quad h_{0}+z^{T} \mu+\|R z\|_{2}+f^{T} w+q^{T} s$

subject to $\quad \underline{u}_{t} \leq \tilde{v}_{t}^{T} \mu-\left\|R \tilde{v}_{t}\right\|_{2}+w_{t}$

$(t=1, \cdots, T)$

$\tilde{v}_{t}^{T} \mu-\left\|R \tilde{v}_{t}\right\|_{2}+w_{t} \leq \bar{u}_{t}$

$(t=1, \cdots, T)$

$\underline{x}_{t} \leq x_{0}+\tilde{l}_{t}^{T}(u-\mu+s)-\Delta d_{t}$

$(t=1, \cdots, T)$ 
$x_{0}+\tilde{l}_{t}^{T}(u-\mu+s)+\Delta d_{t} \leq \bar{x}_{t}$

$(t=1, \cdots, T)$

$s_{t} \geq 0 \quad(t=1, \cdots, T)$

In this model, production quantity should be determined based on the observation of past demand history, thus gives "not too pessimistic" worst case. The problem is a SOCP and thus can be solved efficiently.

\section{NUMERICAL EXAMPLES}

In this section, we give some numerical examples to illustrate the model and variations described before. The data set used in the example is created by modifying the realworld data of an apparel product. The company makes a purchase replenishment order to their supplier within an upper and lower limit of reserved production capacity. All our examples use $T=50$ horizon. Parameters used in the examples are summarized as follows:

- $p_{t}$ : randomly generated using uniform distribution $\mathcal{U}(10,20)$

- $c_{t}=5$ for $t=1, \cdots, T$

- $h_{t}=3$ for $t=1, \cdots, T$

- $(\underline{x}, \bar{x})=(0,300)$ for $t=1, \cdots, T$

- $(\underline{u}, \bar{u})=(20,120)$ for $t=1, \cdots, T$

- $x_{0}=0$

We assume demand has $d_{t}$ is Gaussian random vectors as

$d \sim \mathcal{N}(\mu, \Sigma)$

$\mu_{t}=(1+\cos (2 \pi t / 7)) \times 50$

$\Sigma_{i j}=\left\{\begin{array}{cc}0.3 & i=j \\ 0.4 & i=j+1, i=j-1 \\ 0.1 & i=j+2, i=j-2 \\ 0 & \text { otherwise }\end{array}\right.$

The real time-series data has a strong weekly seasonal pattern, and we modified the data to the seasonal time-series with a seven-period of cycle. We also found that the timeseries data excluding the seasonal pattern can be fitted by the two-dimensional autoregression model, and we use the value of autocorrelation in $\Sigma$.

Our implementation is coded with MATLAB and SeDuMi solver called from CVX, run on Intel Core i7 $3.5 \mathrm{GHz} \times 2,16 \mathrm{~GB}$ memory.

To illustrate the robustness of the proposed model, we solve the following three models
Non-robust model (1a-1e) with $d=\mu$

1. Robust static model

2. Robust dynamic model

We generate 1,000 random demands and compared models by taking $u$ obtained from above optimization with respect to the average and $95 \%$ confidence interval of total cost, the number of infeasible solutions and CPU time in Table 1. From Table 1, we see that the average total cost of both robust models is lower, and the $95 \%$ confidence interval is narrower than the non-robust model. We also see that there are no infeasible solutions in both robust models, whereas the non-robust model gives 9 infeasible solutions. This can be considered as a result of incorporation of robustness in both robust models. Further the dynamic robust model outperforms the other two models. This can be considered as a result of incorporation of dynamic decision-making process in the dynamic robust model. We see that both robust models require less than a second CPU time. This is beneficial in the case where problem must be solved many times, e.g., there are many SKUs.

The cost breakdowns for each of cost items in the objective function for the non-robust model, static robust model and dynamic robust model in Table 2. From Table 2, We see that both robust optimization models have lower lost sales in return for higher inventory cost, in consequence lower total cost than the non-robust model.

In Figure 1 we have plotted the histogram of the distributions of objective functions. Depicted in Figure 1, we see that both robust models are much better in terms of worst-case cost obtained. We also see that robust dynamic model is better in terms of average, best-case, and worst-case cost obtained. This is because of the benefit of dynamic model that the order quantity is determined after observation of past demands. These examples show that effectiveness of applying robust optimization to inventory control problem.

\section{CONCLUSION}

We have considered a problem of finding an optimal set of inventories, production quantity, and shortage quantity as measured by minimum total cost of inventory holding cost, production cost, and lost sales, subject to inventory and order quantity bounds. We have developed an adjustable robust counterpart model that can be easily reduced to the equivalent non-robust problem via second-order cone programming. In the computational experiment, we compared proposed robust models with standard non-robust model and show the proposed model outperforms the nonrobust model.

For future work, we are interested in applying the approximate objective function model with a factor-based demand to explicitly consider correlated demand over time. We are also interested in expanding the model to dynamiclot sizing problem where fixed order cost is incurred.

Table 1 Total cost (average and 95\% trust region for 1,000 times trial), \# of infeasible solutions and CPU time

\begin{tabular}{|l|c|c|c|}
\hline & Total Cost & \# of infeasible solutions & CPU time (sec) \\
\hline Non-robust model & $33,055 \pm 7,325$ & $9 / 1,000$ & 0.05 \\
\hline Static robust model & $31,888 \pm 3,799$ & $0 / 1,000$ & 0.09 \\
\hline Dynamic robust model & $29,945 \pm 2,391$ & $0 / 1,000$ & 0.47 \\
\hline
\end{tabular}


Table 2 Cost breakdowns (average and 95\% trust region of each of cost items for 1,000 times trial)

\begin{tabular}{|l|c|c|c|}
\hline & Production cost & Inventory cost & Lost sales \\
\hline Non-robust model & 24,650 & $2,279 \pm 1,561$ & $6,844 \pm 8,586$ \\
\hline Static robust model & 23,901 & $6,712 \pm 5,167$ & $1,275 \pm 2,978$ \\
\hline Dynamic robust model & 22,176 & $5,329 \pm 3,233$ & $1,440 \pm 1,934$ \\
\hline
\end{tabular}

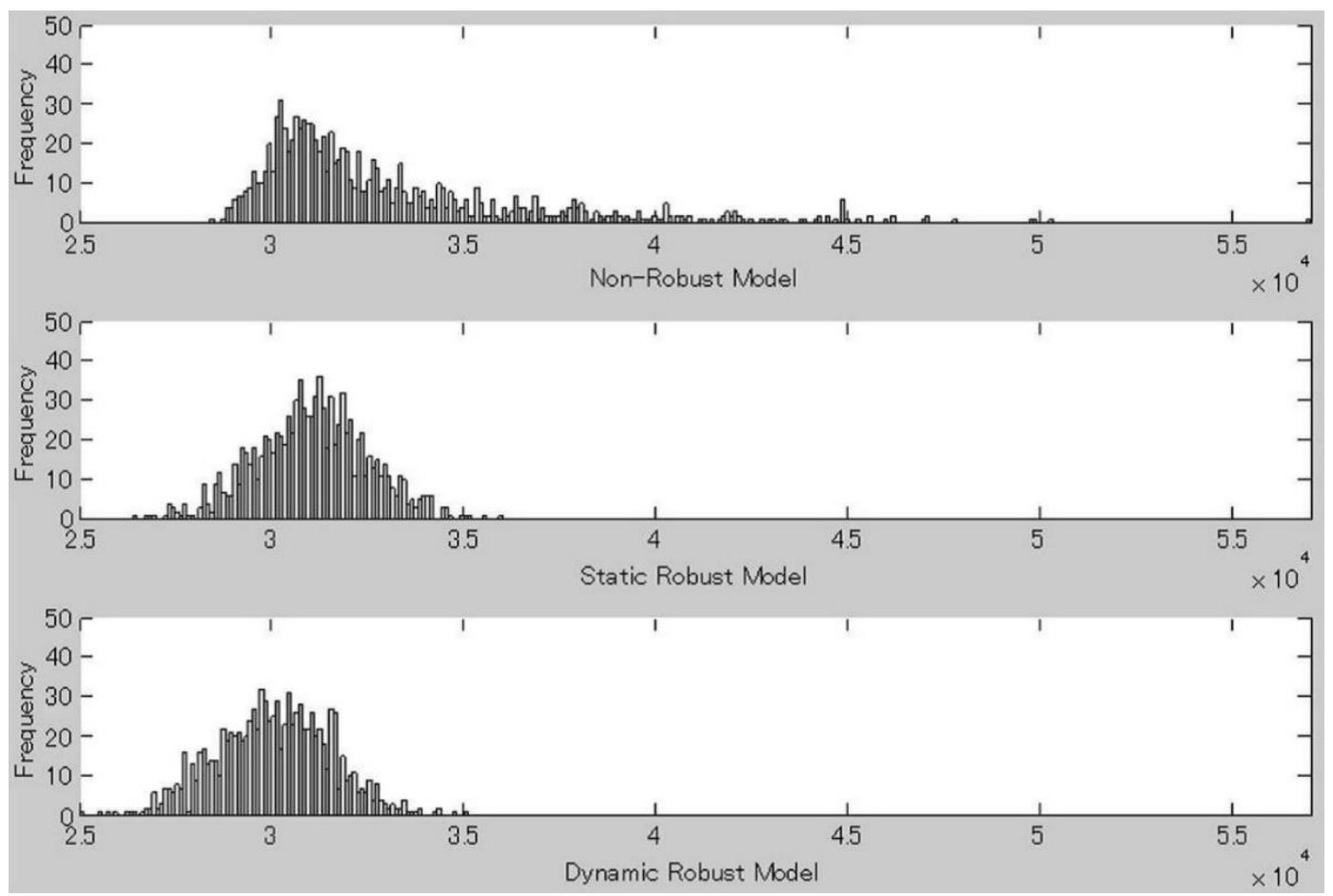

Figure 1 Histogram of the distributions of objective function value (1,000 trials)

\section{REFERENCES}

Aloulou, M. A., Dolgui, A., \& Kovalyov, M. Y. (2014). A bibliography of non-deterministic lot-sizing models. International Journal of Production Research, 52(8), pp. 2293-2310.

Bellman, R., Glicksberg, I., \& Gross, O. (1955). On the optimal inventory equation. Management Science, 2(1), pp. 83-104.

Ben-Tal, A., Boaz, G., \& Shimrit, S. (2009). Robust multiechelon multi-period inventory control. European Journal of Operational Research, 199(3), pp. 922-935.

Ben-Tal, A., Golany, B., Nemirovski, A., \& Vial, J. P. (2005). Retailer-supplier flexible commitments contracts: A robust optimization approach. Manufacturing \& Service Operations Management, 7(3), pp. 248-271.

Ben-Tal, A., Goryashko, A., Guslitzer, E., \& Nemirovski, A. (2004). Adjustable robust solutions of uncertain linear programs. Mathematical programming, 99(2), pp. 351-376.

Bertsimas, D., \& Sim, M. (2003). Robust discrete optimization and network flows. Mathematical programming, 98(1-3), pp. 49-71.

Bertsimas, D., \& Thiele, A. (2004, June). A robust optimization approach to supply chain management. In International Conference on Integer Programming and Combinatorial Optimization (pp. 86-100). Springer, Berlin, Heidelberg.

Bertsimas, D., \& Thiele, A. (2006). A robust optimization approach to inventory theory. Operations research, 54(1), pp. $150-168$.

Bienstock, D., \& ÖZbay, N. (2008). Computing robust basestock levels. Discrete Optimization, 5(2), pp. 389-414.

Bijvank, M., \& Vis, I. F. (2011). Lost-sales inventory theory: A review. European Journal of Operational Research, 215(1), pp. 1-13.
Downs, B., Metters, R., \& Semple, J. (2001). Managing inventory with multiple products, lags in delivery, resource constraints, and lost sales: A mathematical programming approach. Management science, 47(3), pp. 464-479.

Gaver Jr, D. P. (1959). On base-stock level inventory control. Operations Research, 7(6), pp. 689-703.

Gruen, T. W., Corsten, D. S., \& Bharadwaj, S. (2002). Retail outof-stocks: A worldwide examination of extent, causes and consumer responses. Washington, DC: Grocery Manufacturers of America.

Guan, Y., Ahmed, S., Miller, A. J., \& Nemhauser, G. L. (2006). On formulations of the stochastic uncapacitated lot-sizing problem. Operations Research Letters, 34(3), pp. 241-250.

Huh, W. T., Janakiraman, G., Muckstadt, J. A., \& Rusmevichientong, P. (2009). Asymptotic optimality of order-up-to policies in lost sales inventory systems. Management Science, 55(3), pp. 404-420.

Janakiraman, G., \& Roundy, R. O. (2004). Lost-sales problems with stochastic lead times: Convexity results for base-stock policies. Operations Research, 52(5), pp. 795-803.

Johansen, S. G. (2001). Pure and modified base-stock policies for the lost sales inventory system with negligible set-up costs and constant lead times. International Journal of Production Economics, 71(1-3), pp. 391-399.

Karlin, S. (1958). Inventory models of the Arrow-HarrisMarschak type with time lag. Studies in the mathematical theory of inventory and production.

Karlin, S., Scarf, H., \& Arrow, K. J. (1958). Studies in the mathematical theory of inventory and production. Stanford University Press.

Morse, P. M. (1959). Solutions of a class of discrete-time inventory problems. Operations Research, 7(1), pp. 67-78. 
Morton, T. E. (1969). Bounds on the solution of the lagged optimal inventory equation with no demand backlogging and proportional costs. SIAM review, 11(4), pp. 572-596.

Pressman, I. (1977). An order-level-scheduling-period system with lost sales. Management science, 23(12), pp. 13281335.

See, C. T., \& Sim, M. (2010). Robust approximation to multiperiod inventory management. Operations research, 58(3), pp. 583-594.

Solyalı, O., Cordeau, J. F., \& Laporte, G. (2016). The impact of modeling on robust inventory management under demand uncertainty. Management Science, 62(4), pp. 1188-1201.

Tarim, S. A., \& Kingsman, B. G. (2004). The stochastic dynamic production/inventory lot-sizing problem with service-level constraints. International Journal of Production Economics, 88(1), pp. 105-119.

Tempelmeier, H. (2013). Stochastic lot sizing problems. In Handbook of stochastic models and analysis of manufacturing system operations (pp. 313-344). Springer, New York, NY.

Tunc, H., Kilic, O. A., Tarim, S. A., \& Eksioglu, B. (2014). A reformulation for the stochastic lot sizing problem with service-level constraints. Operations Research Letters, 42(2), pp. 161-165.

Underhill, P. (2009). Why we buy: The science of shoppingupdated and revised for the Internet, the global consumer, and beyond. Simon and Schuster.

Van Donselaar, K., De Kok, T., \& Rutten, W. G. M. M. (1996). Two replenishment strategies for the lost sales inventory model: A comparison. International Journal of Production Economics, 46, pp. 285-295.

Zhou, Z., \& Guan, Y. (2013). Two-stage stochastic lot-sizing problem under cost uncertainty. Annals of Operations Research, 209(1), pp. 207-230.

Zipkin, P. (2008). Old and new methods for lost-sales inventory systems. Operations Research, 56(5), pp. 1256-1263.

Shunichi Ohmori (PhD) is an associate professor at Department of Industrial \& System Engineering, Waseda University in Japan, and a researcher at Institute of Global Production \& Logistics at Waseda University, and a researcher at Data Science Institute at Waseda University. He received the master and Ph.D degree in engineering at Waseda University. His research interest lies in operations research and supply chain management.

Kazuho Yoshimoto (Dr.Engg) is a professor at Department of Industrial \& System Engineering at Waseda University in Japan, and a head of Institute of Global Production \& Logistics at Waseda University. He received the master and Ph.D degree in engineering at Waseda University. His research interest lies in facility and logistics design. 